\title{
A GENERATING FUNCTION FOR SUMS OF MULTIPLE ZETA VALUES AND ITS APPLICATIONS
}

\author{
TAKASHI AOKI, YASUHIRO KOMBU, AND YASUO OHNO
}

(Communicated by Jonathan M. Borwein)

\begin{abstract}
A generating function for specified sums of multiple zeta values is defined and a differential equation that characterizes this function is given. As applications, some relations for multiple zeta values over the field of rational numbers are discussed.
\end{abstract}

\section{INTRODUCTION}

There are two types of definitions for multiple zeta values ([1], [6]). For a multiindex $\mathbf{k}=\left(k_{1}, k_{2}, \ldots, k_{n}\right)\left(k_{i} \in \mathbf{N}(i=1,2, \ldots, n), k_{1}>1\right)$, we set

$$
\zeta(\mathbf{k})=\zeta\left(k_{1}, k_{2}, \ldots, k_{n}\right)=\sum_{m_{1}>m_{2}>\cdots>m_{n}>0} \frac{1}{m_{1}^{k_{1}} m_{2} k_{2} \cdots m_{n} k_{n}}
$$

and

$$
\zeta^{*}(\mathbf{k})=\zeta^{*}\left(k_{1}, k_{2}, \ldots, k_{n}\right)=\sum_{m_{1} \geq m_{2} \geq \cdots \geq m_{n} \geq 1} \frac{1}{m_{1} k_{1} m_{2} k_{2} \cdots m_{n} k_{n}} .
$$

The former is normally called a multiple zeta value (MZV for short) and used mainly in the mathematical literatures ([10], [12], [13], [16], [17], etc.). In this article, we are concerned with the latter, which we call a multiple zeta-star value (MZSV for short) to distinguish them from MZVs. Let us remark, however, that the word "multiple zeta values" is sometimes used not only for MZVs but also for MZSVs, for there are Q-linear relations between MZVs and MZSVs, and hence the Q-algebras generated respectively by MZVs and by MZSVs are the same. As is shown in [1] and [15], some part of Q-linear relations that hold among multiple zeta values can be described clearly by using MZSVs. In this article, we introduce a generating function of specified sums of MZSVs and give an ordinary differential equation that characterizes this function. Computing the value of the generating function for unit argument gives some information concerning the relations between MZSVs over Q. This analysis had been done for MZVs in [16]. Our aim is to give an MZSV version of [16]. As a matter of fact, [1] is a part of the answer to this

Received by the editors August 2, 2006.

2000 Mathematics Subject Classification. Primary 11M06, 40B05; Secondary 33C05.

Key words and phrases. Multiple zeta values, hypergeometric functions.

The first author was supported in part by JSPS Grant-in-Aid No. 18540197.

The third author was supported in part by JSPS Grant-in-Aid No. 18540197 and No. 18740020. 
problem. To give the complete answer is not an easy task because we have to get special values of generalized hypergeometric series for the unit argument. In the second half of this article, we will present some partial answers which are obtained under specializations of parameters.

\section{A generating FUnCtion}

For a multi-index $\mathbf{k}=\left(k_{1}, k_{2}, \ldots, k_{n}\right)\left(k_{i} \in \mathbf{N}\right)$, we set

$$
\operatorname{wt}(\mathbf{k})=k_{1}+k_{2}+\cdots+k_{n}, \quad \operatorname{dep}(\mathbf{k})=n, \quad \operatorname{ht}(\mathbf{k})=\#\left\{i \mid k_{i}>1\right\}
$$

and call them the weight, the depth, and the height of $\mathbf{k}$, respectively. The multiindex $\mathbf{k}$ is said to be admissible if $k_{1}>1$. Let $k, n$ and $s$ be nonnegative integers and let $I(k, n, s)$ denote the set of all multi-indices of weight $k$, depth $n$ and of height $s$. We denote by $I_{0}(k, n, s)$ the set of all admissible multi-indices in $I(k, n, s)$. Let $X_{0}(k, n, s ; t)$ denote the sum

$$
X_{0}(k, n, s ; t)=\sum_{\mathbf{k} \in I_{0}(k, n, s)} L_{\mathbf{k}}^{*}(t),
$$

where we set

$$
L_{\mathbf{k}}^{*}(t)=L_{k_{1}, \ldots, k_{n}}^{*}(t)=\sum_{m_{1} \geq m_{2} \geq \cdots \geq m_{n} \geq 1} \frac{t^{m_{1}}}{m_{1}^{k_{1}} m_{2}{ }^{k_{2}} \cdots m_{n}{ }^{k_{n}}} .
$$

The right-hand side of (2.2) converges locally uniformly in the unit disk $|t|<1$ of the complex $t$-plane for every multi-index $\mathbf{k}$. If $\mathbf{k}$ is admissible, then the right-hand side also converges for $t=1$ and the value of it coincides with $\zeta^{*}(\mathbf{k})$. Now we define a generating function $\Phi_{0}^{*}(t)$ for MZSVs by

$$
\Phi_{0}^{*}(t)=\Phi_{0}^{*}(x, y, z ; t)=\sum_{k, n, s} X_{0}(k, n, s ; t) x^{k-n-s} y^{n-s} z^{2 s-2} .
$$

Theorem 2.1. The formal power series $\Phi_{0}^{*}(t)$ is a unique power series solution vanishing at $t=0$ of the differential equation

$$
t^{2}(1-t) \frac{d^{2} w}{d t^{2}}+t\{(1-t)(1-x)-y\} \frac{d w}{d t}+\left(x y-z^{2}\right) w=t .
$$

Hence it converges locally uniformly and defines a holomorphic function in $|t|<1$.

Proof. We define another auxiliary formal power series $\Phi^{*}(t)$ by

$$
\Phi^{*}(t)=\Phi^{*}(x, y, z ; t)=\sum_{k, n, s} X(k, n, s ; t) x^{k-n-s} y^{n-s} z^{2 s},
$$

where we set

$$
X(k, n, s ; t)=\sum_{\mathbf{k} \in I(k, n, s)} L_{\mathbf{k}}^{*}(t) .
$$

Using the formulas

$$
\frac{d}{d t} L_{k_{1}, \ldots, k_{n}}^{*}(t)= \begin{cases}\frac{1}{t} L_{k_{1}-1, k_{2}, \ldots, k_{n}}^{*}(t) & \text { if } \quad k_{1} \geq 2, \\ \frac{1}{t(1-t)} L_{k_{2}, k_{3}, \ldots, k_{n}}^{*}(t) & \text { if } \quad k_{1}=1, n>1\end{cases}
$$


and

$$
\frac{d}{d t} L_{1}^{*}(t)=\frac{1}{1-t}
$$

for the derivative of $L_{\mathbf{k}}^{*}(t)$, we obtain

$$
\begin{gathered}
\frac{d}{d t} X_{0}(k, n, s ; t)=\frac{1}{t}\left(X(k-1, n, s-1 ; t)-X_{0}(k-1, n, s-1 ; t)+X_{0}(k-1, n, s ; t)\right), \\
\frac{d}{d t}\left(X(k, n, s ; t)-X_{0}(k, n, s ; t)\right)=\frac{1}{t(1-t)} X(k-1, n-1, s ; t) .
\end{gathered}
$$

In terms of $\Phi_{0}^{*}(t)$ and $\Phi^{*}(t)$, we find

$\frac{d \Phi_{0}^{*}}{d t}=\frac{1}{y t}\left(\Phi^{*}-1-z^{2} \Phi_{0}^{*}\right)+\frac{x}{t} \Phi_{0}^{*}, \quad \frac{d}{d t}\left(\Phi^{*}-z^{2} \Phi_{0}^{*}\right)=\frac{y}{t(1-t)}\left(\Phi^{*}-1\right)+\frac{y}{1-t}$.

Eliminating $\Phi^{*}$, we see that $w=\Phi_{0}^{*}$ satisfies (2.4). The homogeneous equation of (2.4) is an ordinary differential equation of Fuchsian type with regular singularities at $t=0,1, \infty$, and the characteristic equation at $t=0$ is $\lambda^{2}-(x+y) \lambda+x y-z^{2}=0$. Hence the characteristic exponents at $t=0$ are not positive integers for generic $x, y$, $z$. Therefore (2.4) has a unique formal power series solution in $t$ vanishing at $t=0$ and it should converge locally uniformly in $|t|<1$. This completes the proof.

Although (2.4) is obtained from $\Phi_{0}^{*}$, it can be solved independently of its origin. We shall solve (2.4) in two different ways. First we set $w=\sum_{n=1}^{\infty} a_{n} t^{n}$ and determine $\left\{a_{n}\right\}$ so that $w$ satisfies (2.4). Then we have a recurrence relation for $\left\{a_{n}\right\}$ :

$$
\begin{gathered}
a_{1}=\frac{1}{(1-x)(1-y)-z^{2}}, \\
a_{n+1}=\frac{n(n-x)}{(n+1-x)(n+1-y)-z^{2}} a_{n} \quad(n=1,2, \ldots) .
\end{gathered}
$$

Thus we obtain the following

Proposition 2.2. Let $w$ denote a formal power series

$$
w=\sum_{n=1}^{\infty} a_{n} t^{n}
$$

with

$$
a_{n}=\frac{(n-1) !(1-x)(2-x) \cdots(n-1-x)}{\left((1-x)(1-y)-z^{2}\right)\left((2-x)(2-y)-z^{2}\right) \cdots\left((n-x)(n-y)-z^{2}\right)} .
$$

Then $w$ is a solution to (2.4).

Next we employ the method of variation of constants. Let $\alpha$ and $\beta$ be the characteristic exponents at $t=0$ of the homogeneous equation of (2.4):

$$
\alpha, \beta=\frac{x+y \pm \sqrt{(x-y)^{2}+4 z^{2}}}{2} .
$$

We set

$$
\begin{aligned}
& \varphi_{1}(t)=t^{\alpha} F(\alpha, \alpha-x, \alpha-\beta+1 ; t), \\
& \varphi_{2}(t)=t^{\beta} F(\beta, \beta-x, \beta-\alpha+1 ; t) .
\end{aligned}
$$


Here $F(a, b, c ; t)$ denotes the Gauss hypergeometric function. Then $\left(\varphi_{1}, \varphi_{2}\right)$ is a system of fundamental solutions of the homogeneous equation. By the standard method of solving inhomogeneous differential equation, we have

Proposition 2.3. Let $w$ denote a function

$$
w(t)=u_{1}(t) \varphi_{1}(t)+u_{2}(t) \varphi_{2}(t),
$$

where $u_{1}$ and $u_{2}$ satisfy

$$
\begin{aligned}
& u_{1}^{\prime}(t)=\frac{1}{\alpha-\beta} t^{-\alpha}(1-t)^{y-1} F(\beta, \beta-x, \beta-\alpha+1 ; t), \\
& u_{2}^{\prime}(t)=\frac{1}{\beta-\alpha} t^{-\beta}(1-t)^{y-1} F(\alpha, \alpha-x, \alpha-\beta+1 ; t) .
\end{aligned}
$$

Then $w$ is a solution to (2.4).

Hence if we choose the starting point of integration appropriately to get $u_{1}(t)$ and $u_{2}(t)$, we have the unique holomorphic solution at the origin in the form (2.8). For example, if $\operatorname{Re} \alpha<1$ and $\operatorname{Re} \beta<1$, we set

$$
\begin{aligned}
& u_{1}(t)=\frac{1}{\alpha-\beta} \int_{0}^{t} s^{-\alpha}(1-s)^{y-1} F(\beta, \beta-x, \beta-\alpha+1 ; s) d s, \\
& u_{2}(t)=\frac{1}{\beta-\alpha} \int_{0}^{t} s^{-\beta}(1-s)^{y-1} F(\alpha, \alpha-x, \alpha-\beta+1 ; s) d s .
\end{aligned}
$$

Then we have the holomorphic solution $w$ in the form (2.8). In the sequel, we assume $\operatorname{Re} \alpha<1$ and $\operatorname{Re} \beta<1$ for the sake of simplicity.

\section{Evaluation of THE GENERATING FUNCTION FOR UNIT ARGUMENT}

By the definition of $\Phi_{0}^{*}$, the coefficient of $x^{k-n-s} y^{n-s} z^{2 s-2}$ of its value $\Phi_{0}^{*}(1)$ at $t=1$ is the sum of MZSVs with fixed weight $k$, depth $n$ and height $s$. By using Propositions 2.2, 2.3 and (2.9), (2.10), we may write it in two ways:

$$
\Phi_{0}^{*}(1)=\sum_{n=1}^{\infty} a_{n}
$$

and

$$
\Phi_{0}^{*}(1)=u_{1}(1) \varphi_{1}(1)+u_{2}(1) \varphi_{2}(1) .
$$

Here $u_{1}(1), u_{2}(1)$ are given by

$$
\begin{aligned}
& u_{1}(1)=\frac{1}{\alpha-\beta} \int_{0}^{1} s^{-\alpha}(1-s)^{y-1} F(\beta, \beta-x, \beta-\alpha+1 ; s) d s, \\
& u_{2}(1)=\frac{1}{\beta-\alpha} \int_{0}^{1} s^{-\beta}(1-s)^{y-1} F(\alpha, \alpha-x, \alpha-\beta+1 ; s) d s
\end{aligned}
$$

and $\varphi_{1}(1), \varphi_{2}(1)$ are evaluated by the Gauss formula $[5$, p. 104, (46)]:

$$
\begin{aligned}
& \varphi_{1}(1)=\frac{\Gamma(\alpha-\beta+1) \Gamma(x-\alpha-\beta+1)}{\Gamma(1-\beta) \Gamma(x-\beta+1)}, \\
& \varphi_{2}(1)=\frac{\Gamma(\beta-\alpha+1) \Gamma(x-\alpha-\beta+1)}{\Gamma(1-\alpha) \Gamma(x-\alpha+1)} .
\end{aligned}
$$


Thus we see that $\Phi_{0}^{*}(1)$ has the value

$$
\begin{array}{r}
\int_{0}^{1} d s \quad s^{-\beta}(1-s)^{y-1}\left\{\frac{\Gamma(\beta-\alpha) \Gamma(x-\alpha-\beta+1)}{\Gamma(1-\alpha) \Gamma(x-\alpha+1)} F(\alpha, \alpha-x, \alpha-\beta+1 ; s)\right. \\
\left.+\frac{\Gamma(\alpha-\beta) \Gamma(x-\alpha-\beta+1)}{\Gamma(1-\beta) \Gamma(x-\beta+1)} s^{\beta-\alpha} F(\beta, \beta-x, \beta-\alpha+1 ; s)\right\} .
\end{array}
$$

Using one of the connection formulas for the Gauss hypergeometric functions (e.g., $[5$, p. $108,(43)])$, we rewrite this into the following form:

$$
\frac{1}{1-y} \int_{0}^{1} s^{-\beta} F(1-\beta, 1-\beta+x, 2-y ; 1-s) d s .
$$

Hence we have

Proposition 3.1. Under the notation as above, the following equality holds:

$$
\Phi_{0}^{*}(1)=\frac{1}{1-y} \int_{0}^{1}(1-t)^{-\beta} F(1-\beta, 1-\beta+x, 2-y ; t) d t .
$$

Remark 3.2. Taking term-by-term integration in the right-hand side of (3.7), we can rewrite it in the form

$$
\frac{1}{(1-y)(1-\beta)}{ }_{3} F_{2}(1-\beta, 1-\beta+x, 1 ; 2-y, 2-\beta ; 1),
$$

where ${ }_{3} F_{2}\left(a, b, c ; a^{\prime}, b^{\prime} ; t\right)$ denotes the generalized hypergeometric function [5, p.182]:

$$
{ }_{3} F_{2}\left(a, b, c ; a^{\prime}, b^{\prime} ; t\right)=\frac{\Gamma\left(a^{\prime}\right) \Gamma\left(b^{\prime}\right)}{\Gamma(a) \Gamma(b) \Gamma(c)} \sum_{n=0}^{\infty} \frac{\Gamma(a+n) \Gamma(b+n) \Gamma(c+n)}{\Gamma\left(a^{\prime}+n\right) \Gamma\left(b^{\prime}+n\right) n !} t^{n} .
$$

\section{Specializations of PARAmeters}

In some special cases, we can evaluate $\Phi_{0}^{*}(1)$ in terms of well known quantities.

4.1. The case where $y=x$. The case where $y=x$ in (3.1) is investigated in [1]. We can evaluate the sum $\sum_{n=1}^{\infty}\left(\left.a_{n}\right|_{y=x}\right)$ by using the Gauss formula:

$$
\sum_{n=1}^{\infty}\left(\left.a_{n}\right|_{y=x}\right)=\frac{1}{z} \sum_{l=1}^{\infty}(-1)^{l}\left(\frac{1}{x+z-l}-\frac{1}{x-z-l}\right) .
$$

Comparing the coefficient of $x^{k-2 s} z^{2 s-2}$ of $\left.\Phi_{0}^{*}(1)\right|_{y=x}$ with that of the right-hand side of (4.1), we have

$$
\sum_{\mathbf{k} \in I_{0}(k, *, s)} \zeta^{*}(\mathbf{k})=2\left(\begin{array}{c}
k-1 \\
2 s-1
\end{array}\right)\left(1-2^{1-k}\right) \zeta(k),
$$

which is the main result of [1]. Here we set $I_{0}(k, *, s)=\bigcup_{n} I_{0}(k, n, s)$. Setting $x=0$ in (4.1), we have the generating function for $\zeta^{*}\left(\{2\}_{s}\right):=\zeta^{*}(\underbrace{2, \ldots, 2}_{s})$ :

$$
\sum_{s=1}^{\infty} \zeta^{*}\left(\{2\}_{s}\right) z^{2 s-2}=2 \sum_{l=1}^{\infty} \frac{(-1)^{l}}{z^{2}-l^{2}} .
$$

Taking the expansion of the right-hand side in $z[18,(2.2 .1)]$, we have

$$
2 \sum_{s=1}^{\infty}\left(1-2^{1-2 s}\right) \zeta(2 s) z^{2 s-2} \text {. }
$$


Thus we have the explicit form of $\zeta^{*}\left(\{2\}_{s}\right)$ :

$$
\zeta^{*}\left(\{2\}_{s}\right)=2\left(1-2^{1-2 s}\right) \zeta(2 s),
$$

or equivalently,

$$
\zeta^{*}\left(\{2\}_{s}\right)=(-1)^{s-1} 2\left(2^{2 s-1}-1\right) \frac{B_{2 s}}{(2 s) !} \pi^{2 s},
$$

where $B_{m}$ denote the Bernoulli numbers:

$$
\frac{t e^{t}}{e^{t}-1}=\sum_{m=0}^{\infty} B_{m} \frac{t^{m}}{m !}
$$

Remark 4.1. M. Kaneko has pointed out that (4.6) can also be obtained in an elementary way. That is, computing the expansion of

$$
\frac{\pi z}{\sin \pi z}=\prod_{m=1}^{\infty}\left(1-\frac{z^{2}}{m^{2}}\right)^{-1}
$$

yields (4.6). This function is clearly equal to the right-hand side of (4.3) up to some trivial factors. Note that

$$
\frac{\sin \pi z}{\pi z}=\prod_{m=1}^{\infty}\left(1-\frac{z^{2}}{m^{2}}\right)
$$

is a generating function for $\zeta\left(\{2\}_{s}\right)$.

4.2. The case where $z^{2}=x y$. Let us consider the case where $z^{2}=x y$. Since $\alpha \beta=0$, we may assume $\alpha=0$ and hence $\beta=x+y$. It follows from (3.7) that

$$
\left.\Phi_{0}^{*}(1)\right|_{z^{2}=x y}=\frac{1}{1-y} \int_{0}^{1}(1-t)^{-x-y} F(1-x-y, 1-y, 2-y ; t) d t .
$$

Replacing $F$ by its corresponding series expression and taking term-by-term integration yield

$$
\sum_{l=0}^{\infty} \frac{(1-x-y)_{l}}{l !(l+1-y)} \int_{0}^{1}(1-t)^{-x-y} t^{l} d t=\frac{1}{x} \sum_{l=1}^{\infty}\left(\frac{1}{l-x-y}-\frac{1}{l-y}\right) .
$$

The Taylor expansion of the right-hand side with respect to $x, y$ can be computed easily:

$$
\frac{1}{x} \sum_{k, n} \frac{(k-1) !}{(k-n) !(n-1) !}\left(\sum_{l} \frac{1}{l^{k}}\right) x^{k-n} y^{n-1}=\sum_{k, n}\left(\begin{array}{l}
k-1 \\
n-1
\end{array}\right) \zeta(k) x^{k-n-1} y^{n-1} .
$$

Comparing the coefficient of $x^{k-n-1} y^{n-1}$ with that of $\left.\Phi_{0}^{*}(1)\right|_{z^{2}=x y}$, which is equal to $\sum_{\mathbf{k} \in I_{0}(k, n)} \zeta^{*}(\mathbf{k})$, we have

$$
\sum_{\mathbf{k} \in I_{0}(k, n, *)} \zeta^{*}(\mathbf{k})=\left(\begin{array}{c}
k-1 \\
n-1
\end{array}\right) \zeta(k) .
$$

Here we set $I_{0}(k, n, *)=\bigcup_{s} I_{0}(k, n, s)$. Thus we have obtained a new proof of the sum formula for MZSVs ([11]). See [15] for another proof of it. We refer the reader to [8] for the equivalence of the sum formula of MZVs and that of MZSVs. See also [7], [20] for the proof of the sum formula for MZVs. 
4.3. The case where $y=0$. Next we set $y=0$. This specialization gives the generating function of MZSVs with full height. The coefficients of the function are the collections of MZSVs $\zeta^{*}(\mathbf{k})$ for which every component of multi-index $\mathbf{k}$ is greater than or equal to 2 . We write

$$
\Phi_{0}^{*}(x, z)=\left.\Phi_{0}^{*}(1)\right|_{y=0}=\sum_{k, n} X_{0}(k, n, n ; 1) x^{k-2 n} z^{2 n-2}
$$

and evaluate it by using (3.7).

Theorem 4.2. The power series (4.9) is given by

$$
\Phi_{0}^{*}(x, z)=-\frac{1}{z^{2}}\left(1-\exp \left(\sum_{n=2}^{\infty} \frac{\zeta(n)}{n} S_{n}(x, z)\right)\right),
$$

where the polynomials $S_{n}(x, z) \in \mathbf{Z}[x, z]$ are defined by the formula

$$
S_{n}(x, z)=\alpha^{n}+\beta^{n}-x^{n}, \quad \text { where } \alpha, \beta=\frac{x \pm \sqrt{x^{2}+4 z^{2}}}{2}
$$

or alternatively by the identity

$$
\log \left(1+\frac{z^{2}}{1-x-z^{2}}\right)=\sum_{n=1}^{\infty} \frac{S_{n}(x, z)}{n}
$$

together with the requirement that $S_{n}(x, z)$ is a homogeneous polynomial of degree $n$. In particular, all of the coefficients $X_{0}(k, n, n ; 1)$ of $\Phi_{0}^{*}(x, z)$ can be expressed as polynomials in $\zeta(2), \zeta(3), \ldots$ with rational coefficients.

Proof. Since $\alpha+\beta=x$ and $\alpha \beta=-z^{2}$, it follows from (3.7) that

$$
\Phi_{0}^{*}(x, z)=\int_{0}^{1}(1-t)^{-\beta} F(1-\beta, 1+\alpha, 2 ; t) d t
$$

holds. Term-by-term integration in the right-hand side and the Gauss formula yield

$$
\begin{aligned}
\Phi_{0}^{*}(x, z) & =\sum_{k=0}^{\infty} \frac{(1-\beta)_{k}(1+\alpha)_{k}}{(k+1) ! k !} \int_{0}^{1}(1-t)^{-\beta} t^{k} d t \\
& =\sum_{k=0}^{\infty} \frac{(1+\alpha)_{k}}{(k+1) !(1-\beta+k)} \\
& =\frac{1}{\alpha \beta}\left(1-\frac{\Gamma(1-\alpha) \Gamma(1-\beta)}{\Gamma(1-\alpha-\beta)}\right) .
\end{aligned}
$$

Combining this with the expansion

$$
\Gamma(1-x)=\exp \left(\gamma x+\sum_{n=2}^{\infty} \frac{\zeta(n)}{n} x^{n}\right),
$$

we obtain (4.10). 
4.4. The case where $z=0$. Finally we consider the case where $z=0$. We use (3.1) to evaluate $\left.\Phi_{0}^{*}(1)\right|_{z=0}$. Since we have, by a partial fraction decomposition,

$$
\begin{aligned}
\left.a_{n}\right|_{z=0} & =\frac{(n-1) !}{(n-x)(1-y)(2-y) \cdots(n-y)} \\
& =\sum_{m=1}^{n} \frac{(-1)^{m-1}(n-1) !}{(m-1) !(n-m) !(n-x)(m-y)},
\end{aligned}
$$

we can compute the Taylor expansion of $\left.\sum a_{n}\right|_{z=0}$ in $x, y$ :

$$
\sum_{n=1}^{\infty}\left(\left.a_{n}\right|_{z=0}\right)=\sum_{i, j=1}^{\infty} \sum_{n \geq m \geq 1}(-1)^{m-1}\left(\begin{array}{c}
n-1 \\
m-1
\end{array}\right) \frac{1}{n^{i} m^{j}} x^{i-1} y^{j-1} .
$$

Noting that

$$
\left.\Phi_{0}^{*}(1)\right|_{z=0}=\sum_{k, n} X_{0}(k, n, 1) x^{k-n-1} y^{n-1}
$$

and

$$
X_{0}(k, n, 1)=\zeta^{*}\left(k-n+1,\{1\}_{n-1}\right):=\zeta^{*}(k-n+1, \underbrace{1, \ldots, 1}_{n-1}),
$$

we have the following expression:

Proposition 4.3. Let $i$ and $j$ be positive integers. Then

$$
\zeta^{*}\left(i+1,\{1\}_{j-1}\right)=\sum_{n \geq m \geq 1}(-1)^{m-1}\left(\begin{array}{c}
n-1 \\
m-1
\end{array}\right) \frac{1}{n^{i} m^{j}}
$$

holds.

Remark 4.4. The left-hand side of (4.15) is $\xi_{j}(i)$ of Arakawa-Kaneko [2] (see [1] also).

Remark 4.5. Comparing the definition of MZSVs with the right-hand side of (4.15), we observe that the following equality should hold for every fixed $n$ and $j$ :

$$
\sum_{n \geq m_{1} \geq m_{2} \geq \cdots \geq m_{j-1} \geq 1} \frac{1}{m_{1} m_{2} \cdots m_{j-1}}=\sum_{m=1}^{n}(-1)^{m-1}\left(\begin{array}{c}
n \\
m
\end{array}\right) \frac{1}{m^{j-1}} .
$$

This identity appears in Corollary 3 of [4].

Remark 4.6. A generating function for $\zeta\left(m+2,\{1\}_{n}\right)$ is given in [3], [20]:

$$
\sum_{m, n \geq 1} x^{m+1} y^{n+1} \zeta\left(m+2,\{1\}_{n}\right)=1-\exp \left(\sum_{k \geq 2} \frac{x^{k}+y^{k}-(x+y)^{k}}{k} \zeta(k)\right) .
$$

\section{ACKNOWLEDGMENTS}

The authors express their sincere gratitude to Professor M. Kaneko for useful discussions and invaluable advice, especially for the content of Remark 4.1. 


\section{REFERENCES}

1. T. Aoki and Y. Ohno, Sum relations for multiple zeta values and connection formulas for the Gauss hypergeometric function, Publ. RIMS, Kyoto Univ. 41 (2005), 329-337. MR2138027 (2005m:11165)

2. T. Arakawa and M. Kaneko, Multiple zeta-values, poly-Bernoulli numbers, and related zeta functions, Nagoya Math. J. 153 (1999), 1-21. MR1684557 (2000e:11113)

3. J. Borwein, D. Bradley and D. Broadhurst, Evaluations of $k$-fold Euler/Zagier sums: a compendium of results for arbitrary $k$. The Wilf Festschrift Volume. Electron. J. Combin. 4 (1997), no. 2, Research Paper 5, 19 pp. MR1444152 (98b:11091)

4. K. Dilcher, Some $q$-series identities related to divisor functions, Disc. Math. 145 (1995), 83-93. MR1356587 (96i:11020)

5. A. Erdélyi et al. (eds.), Higher Transcendental Functions, vol. 1, Robert E. Krieger Publishing Company, Malabar, 1985.

6. L. Euler, Meditationes circa singulare serierum genus, Novi Comm. Acad. Sci. Petropol 20 (1775), 140-186, reprinted in Opera Omnia ser. I, vol. 15, B. G. Teubner, Berlin (1927), $217-267$.

7. A. Granville, A decomposition of Riemann's zeta-function, in Analytic Number Theory, London Mathematical Society Lecture Note Series 247, Y. Motohashi (ed.), Cambridge University Press, (1997), 95-101. MR1694987 (2000c:11134)

8. M. Hoffman, Multiple harmonic series, Pacific J. Math. 152 (1992), 275-290. MR1141796 (92i:11089)

9. M. Hoffman, Algebraic aspects of multiple zeta values, In Zeta Functions, Topology and Quantum Physics (T. Aoki, S. Kanemitsu, M. Nakahara and Y. Ohno, eds.), Developments in Mathematics 14, Springer, 2005, pp. 51-74. MR2179272 (2006g:11185)

10. M. Hoffman and Y. Ohno, Relations of multiple zeta values and their algebraic expression, $J$. Algebra 262 (2003), 332-347. MR1971042 (2004c:11163)

11. Y. Kombu, Multiple zeta values and hypergeometric differential equations (in Japanese), Kinki University master's thesis (2003).

12. T. Q. T. Le and J. Murakami, Kontsevich's integral for the Homfly polynomial and relations between values of multiple zeta functions, Topology and its Applications 62 (1995), 193-206. MR1320252 (96c:57017)

13. Y. Ohno, A generalization of the duality and sum formulas on the multiple zeta values. $J$. Number Theory 74 (1999), 39-43. MR1670544 (99k:11138)

14. Y. Ohno, Sum relations for multiple zeta values. In Zeta Functions, Topology and Quantum Physics (T. Aoki, S. Kanemitsu, M. Nakahara and Y. Ohno eds.), Developments in Mathematics 14, Springer, 2005, pp. 131-144. MR2179276 (2006i:11105)

15. Y. Ohno and N. Wakabayashi, Cyclic sum of multiple zeta values, Acta Arithmetica 123 (2006), 289-295. MR2263259 (2007g:11113)

16. Y. Ohno and D. Zagier, Multiple zeta values of fixed weight, depth, and height, Indag. Math. 12 (2001), 483-487. MR1908876 (2003e:11094)

17. J. Okuda and K. Ueno, Relations for multiple zeta values and Mellin transforms of multiple polylogarithms. Publ. RIMS, Kyoto Univ. 40 (2004), 537-564. MR2049646 (2005f:11199)

18. E. C. Titchmarsh, The Theory of the Riemann Zeta-Function, 2nd edition revised by D. R. Heath-Brown, Oxford University Press, Oxford, 1986. MR882550 (88c:11049)

19. D. Zagier, Values of zeta functions and their applications. In Proceedings of ECM 1992, Progress in Math. 120 (1994), 497-512. MR1341859 (96k:11110)

20. D. Zagier, Multiple zeta values. Unpublished manuscript, Bonn, 1995.

Department of Mathematics, Kinki University, Higashi-Osaka, Osaka 577-8502, Japan E-mail address: aoki@math.kindai.ac.jp

Department of Mathematics, Kinki University, Higashi-Osaka, Osaka 577-8502, Japan E-mail address: kombu@math.kindai.ac.jp

Department of Mathematics, Kinki University, Higashi-Osaka, Osaka 577-8502, Japan

E-mail address: ohno@math.kindai.ac.jp 